\title{
Relative contributions of brain and cervical cord pathology to multiple sclerosis disability: a study with magnetisation transfer ratio histogram analysis
}

Marco Rovaris, Marco Bozzali, Giuseppe Santuccio, Giuseppe Iannucci, Maria Pia Sormani, Bruno Colombo, Giancarlo Comi, Massimo Filippi

\begin{abstract}
Objective-To assess (a) the correlations between magnetisation transfer ratio (MTR) histogram derived measures of the brain and the cervical cord from patients with different multiple sclerosis phenotypes and (b) the correlation between these metrics and clinical disability. Magnetisation transfer imaging is sensitive to the most destructive aspects of multiple sclerosis pathology. Magnetisation transfer ratio histogram analysis encompasses the macroscopic and the microscopic lesion burdens.
\end{abstract}

Methods-Seventy seven patients with multiple sclerosis were studied (40 relapsing-remitting ( $R R$ ), 28 secondary progressive $(\mathrm{SP})$, and nine primary progressive (PP)). For the brain, we obtained dual echo, T1 weighted, and gradient echo (GE) scans (with and without an MT saturation pulse). For the cervical cord, fast short tau inversion recovery (STIR) and GE scans (with and without an MT saturation pulse) were obtained. Brain T2 and $\mathrm{T} 1$ weighted lesion volumes (LVs) were measured. The number and length of cord lesions on fast STIR scans were assessed. Magnetisation transfer ratio maps were created from GE images and MTR histograms of the entire brain and cervical cord were obtained.

Neuroimaging

Research Unit,

Department of

Neuroscience,

Scientific Institute

Ospedale San Raffaele,

University of Milan,

Via Olgettina 60, 20132

Milan, Italy

M Rovaris

M Bozzali

G Santuccio

G Iannucci

M P Sormani

M Filippi

Clinical Trials Unit B Colombo

G Comi

Correspondence to: Dr Massimo Filippi

filippi.massimo@hsr.it

Received 22 February 2000 and in revised form

11 May 2000

Accepted 6 June 2000
Results-Brain T1 LV, and number and size of cord lesions were significantly higher and brain MTR histogram peak location was significantly lower in patients with SPMS than those with RRMS or PPMS. Cord MTR histogram peak location was also significantly lower in patients with SPMS than in those with RRMS. The univariate correlations between MTR histogram derived metrics obtained from the brain and the cervical cord were all non-significant, with the exception of that between average brain MTR and cord MTR histogram peak location. On a multivariable analysis, both increasing brain $T 2 L V$ and decreasing cord MTR histogram peak location values were significantly associated with a higher probability for patients to have SPMS or to have locomotor disability.

Conclusions-This study shows that the extent and severity of tissue damage in the brain and cervical cord are both relevant to determine disability in multiple sclerosis and that the assessment of brain and cord pathology provides complementary information.

(F Neurol Neurosurg Psychiatry 2000;69:723-727)

Keywords: multiple sclerosis; magnetic resonance imaging; magnetisation transfer imaging; brain; cervical cord; histogram analysis

Conventional magnetic resonance imaging of the brain is highly sensitive for detecting focal lesions in patients with multiple sclerosis. ${ }^{1}$ This is why assessment of the burden of brain T2 lesions is used to monitor evolution of multiple sclerosis, either natural or modified by treatment. ${ }^{2}$ However, the magnitude of the correlation between brain MRI lesion load and clinical disability is only modest. ${ }^{2}$ Spinal cord MRI is less sensitive than brain MRI for detecting multiple sclerosis lesions, ${ }^{4-7}$ but there is evidence that the occurrence of multiple sclerosis lesions in the cord gives rise to clinical symptoms more often than lesions located in the brain. ${ }^{8}{ }^{9}$ In addition, although patients with the progressive forms of multiple sclerosis may have brain T2 lesion burdens which overlap with those of patients with less disabling disease courses, ${ }^{3}$ they tend to have more cord abnormalities. ${ }^{5} 10$

The assessment of multiple sclerosis pathology using T 2 weighted images is limited by the poor specificity of this technique to the heterogeneous substrates of multiple sclerosis lesions ${ }^{11}$ and by its inability to provide information about the microscopic changes occurring in the normal appearing white matter. ${ }^{12}$ Although hypointense lesions on T1 weighted images are those in which severe tissue loss has occurred, ${ }^{13}{ }^{14}$ the assessment of their burden ${ }^{13}$ does not allow the quantification of the severity of intrinsic lesion pathology and does not give any information about damage to normal appearing white matter. Other MR techniques, including magnetisation transfer imaging (MTI), ${ }^{15}$ go some way towards overcoming these limitations. Magnetisation transfer imaging provides quantitative metrics which are sensitive to the most destructive aspects of multiple sclerosis pathology ${ }^{16} 17$ and allows the macroscopic and microscopic disease burdens to be estimated. ${ }^{18}$ Low MT ratio (MTR) indicates a reduced capacity of the molecules in the brain tissue matrix to 
exchange magnetisation with the surrounding water molecules and, in multiple sclerosis, this has been shown to be strongly correlated with the percentage of residual myelin and axons present in lesions and normal appearing white matter. ${ }^{19}$ In addition, MTR histogram analy$\operatorname{sis}^{20}$ encompasses both the macroscopic and the microscopic multiple sclerosis pathology. Several studies have shown that MTR histogram derived measures of the brain ${ }^{31-23}$ and cervical cord $^{10}$ correlate well with the clinical manifestations of multiple sclerosis.

Whereas other studies ${ }^{3021-23}$ assessed the burden of multiple sclerosis pathology either in the brain or in the cervical cord of patients with multiple sclerosis, the relation between the presence and extent of multiple sclerosis damage in these two regions has not been investigated. Moreover, their relative contributions to the development of irreversible, multiple sclerosis related disability are still unclear. Aims of this study were: $(a)$ to evaluate the amount of the macroscopic and microscopic tissue damage in a large portion of the CNS of patients with different multiple sclerosis phenotypes, (b) to investigate the magnitude of the correlation between MTR histogram derived measures of the brain and the cervical cord, and $(c)$ to investigate the magnitude of the correlation between brain and cervical cord MTR histogram measures and clinical disability.

\section{Materials and methods}

PATIENTS

Patients included had had clinically definite multiple sclerosis ${ }^{24}$ for at least two years. They were classified as relapsing-remitting (RR) multiple sclerosis (clearly defined disease relapses with either full recovery or sequelae, but without disease progression during the periods between the relapses), secondary progressive (SP) multiple sclerosis (initial relapsing-remitting course followed by progression with or without occasional relapses, minor remissions, or plateaux), or primary progressive (PP) multiple sclerosis (disease progression from onset with occasional plateaux and temporary minor improvements). ${ }^{25}$ None of the patients had either relapses or steroid treatments during the 3 months before study entry. At the time MRI was performed, patients were assessed neurologically by a single observer, unaware of the MRI results, and disability was scored using the expanded disability status scale (EDSS) ${ }^{26}$ Local ethics committee approval and written informed consent were obtained from all the patients before study initiation.

\section{IMAGE ACQUISITION}

Using a 1.5 Tesla system (Vision, Siemens, Erlangen, Germany), the following pulse sequences were acquired for brain imaging:

(a) dual echo turbo spin echo (TSE) ( $\mathrm{TR}=3300, \mathrm{TE}=16 / 98$, echo train length=5); (b) $2 \mathrm{D}$ gradient echo $(\mathrm{GE})(\mathrm{TR}=640, \mathrm{TE}=12$, flip angle $=20^{\circ}$ ), both with and without an MT saturation pulse (this was an off resonance radio frequency pulse centred $1.5 \mathrm{kHz}$ below the water frequency with a gaussian envelope duration of $7.68 \mathrm{~ms}$ and a flip angle of $500^{\circ}$ ); (c) T1 weighted conventional spin echo ( $\mathrm{TR}=768, \mathrm{TE}=14)$. Twenty four contiguous, axial slices with $5 \mathrm{~mm}$ thickness, $192 \times 256$ matrix size, and $188 \times 250 \mathrm{~mm}$ field of view (FOV) were obtained. Scan positioning was performed according to published guidelines. ${ }^{27}$

In the same scanning session, the following pulse sequences were acquired for cervical cord imaging, using a tailored cervical cord phased array coil for signal reception: (a) fast short tau inversion recovery (fast STIR) $(\mathrm{TR}=2288$, $\mathrm{TE}=60$, $\mathrm{TI}=110$, echo train length $=11$, FOV $=280 \times 280 \mathrm{~mm}$, matrix size $=264 \times 512)$; (b) $2 \mathrm{D}$ GE $\left(\mathrm{TR}=640, \mathrm{TE}=10\right.$, flip angle $=20^{\circ}$, FOV $=250 \times 250 \mathrm{~mm}$, matrix size $=192 \times 256$ ), both with and without an MT saturation pulse (the saturation pulse had the same characteristics as that used for brain imaging). For the fast STIR scans, eight interleaved, sagittal slices with a thickness of $3 \mathrm{~mm}$ and an interslice gap of $0.3 \mathrm{~mm}$ were obtained. For the GE scans, 20 contiguous interleaved, axial slices with a thickness of $5 \mathrm{~mm}$ were obtained.

IMAGE ANALYSIS

According to published guidelines, ${ }^{3}$ two experienced observers, without knowing to whom the scans belonged, identified by consensus the hyperintense lesions on proton density (PD) weighted and the hypointense lesions on T1 weighted scans. T2 weighted images were always used to increase confidence in lesion identification. On both PD weighted and T1 weighted images, lesions were marked on the hard copies and lesion volumes (LVs) measured by a single observer, unaware of the patients' clinical characteristics. A local thresholding technique was used for lesion segmentation, keeping the marked hard copies as a reference. Further details about this image analysis method have been extensively reported elsewhere. ${ }^{28}$ On the hard copies of the fast STIR scans, multiple sclerosis lesions in the cervical cord were identified by agreement by the same two observers, without knowing to whom the scans belonged. The length of each lesion was measured by counting the number of vertebral segments corresponding to it.

Brain and cervical cord MTR histograms were obtained as follows. Firstly, the two GE images (with and without the MT saturation pulse) were coregistered. Registration was performed using an automated technique based on pixel similarity measures. ${ }^{29}$ Next, an MTR image was calculated from the coregistered GE images according to the following formula:

$\operatorname{MTR}=\left(M_{0}-M_{s}\right) / M_{0} \times 100$

in which $M_{0}$ is the signal intensity for a given pixel without the saturation pulse and $M_{s}$ is the signal intensity for the same pixel when the saturation pulse is applied. Then the entire brain and cervical cord tissue were segmented from the MTR images by one observer, without knowing to whom the scans belonged, using a segmentation technique based on local thresholding. ${ }^{28}$ Finally, MTR histograms (with bins $1 \%$ in width) were created. We excluded from the analysis all the pixels with MTR values lower than $10 \%$, to minimise partial vol- 
Table 1 Brain T2 and T1 lesion volumes and number and length of cervical cord lesions from the whole patient sample and the three multiple sclerosis (MS) phenotypes

\begin{tabular}{llllll}
\hline & All patients & RRMS & SPMS & PPMS & p Value \\
\hline Brain T2 LV (ml) & $15.3(15.5)$ & $11.4(9.1)$ & $22.2(20.8)$ & $8.0(5.9)$ & 0.08 \\
Brain T1 LV (ml) & $5.3(5.1)$ & $3.4(3.3)$ & $6.4(3.9)$ & $0.9(0.7)$ & 0.03 \\
Number of cord lesions & $2.0(1.4)$ & $1.6(1.3)$ & $2.8(1.3)$ & $1.5(1.0)$ & 0.002 \\
Length of cord lesions & $3.3(2.5)$ & $2.5(1.0)$ & $4.7(2.8)$ & $2.5(1.9)$ & 0.004 \\
\hline
\end{tabular}

Values are expressed as means (SD). LV=Lesion volume; RRMS=relapsing-remitting $M S$; SPMS=secondary progressive MS; PPMS=primary progressive MS. For statistical analysis, see text.

Table 2 Magnetisation transfer ratio histogram derived metrics of the brain and cervical cord from the whole patient sample and the three multiple sclerosis (MS) phenotypes

\begin{tabular}{llllll}
\hline & All patients & \multirow{2}{*}{ RRMS } & \multirow{2}{*}{ SPMS } & PPMS & p Value \\
\hline Average MTR: brain (\%) & $39.2(1.3)$ & $39.4(1.6)$ & $38.7(1.0)$ & $39.7(0.6)$ & NS \\
Peak height: brain & $103.8(14.9)$ & $106.0(16.2)$ & $100.0(14.1)$ & $105.9(8.4)$ & NS \\
Peak location: brain (\%) & $34.4(1.7)$ & $34.6(1.6)$ & $33.6(1.7)$ & $35.4(1.0)$ & 0.007 \\
Average MTR: cord (\%) & $44.4(2.4)$ & $44.9(2.3)$ & $43.8(2.5)$ & $43.7(1.5)$ & NS \\
Peak height: cord & $63.9(15.2)$ & $66.5(17.5)$ & $60.9(11.1)$ & $61.8(15.1)$ & NS \\
Peak location: cord (\%) & $39.1(3.4)$ & $40.2(3.1)$ & $38.1(3.3)$ & $37.3(4.0)$ & 0.01
\end{tabular}

Values are expressed as means (SD). MTR=Magnetisation transfer ratio; RRMS=relapsingremitting MS; SPMS=secondary progressive MS; PPMS=primary progressive $M S$. For statistical analysis, see text.

ume effects from CSF. To correct for the between patient differences in brain and cord volumes, each histogram was normalised by dividing it by the total number of pixels included. For each histogram, the following measures were derived: the average MTR, the peak height (proportion of pixels at the most common MTR value), and the peak position (the most common MTR). All histogram derived measures were from the whole of the brain and cervical cord tissue, thus including both visible lesions and normal appearing tissue.

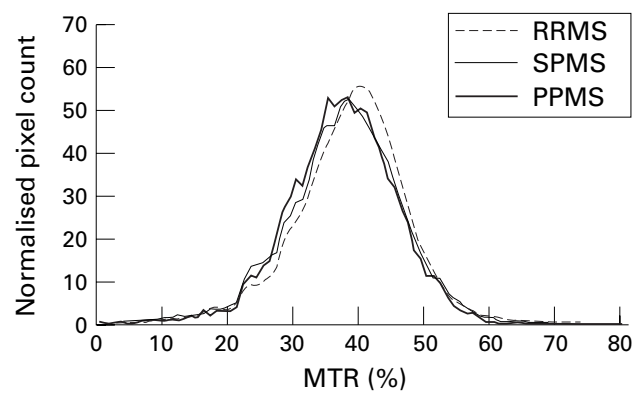

Figure 1 Average MTR histograms of the cervical cord from RRMS (dotted line), SPMS (grey line), and PPMS (black line) patient groups. The shape of the histograms almost overlaps for SPMS and PPMS patient groups. See the text for further details.
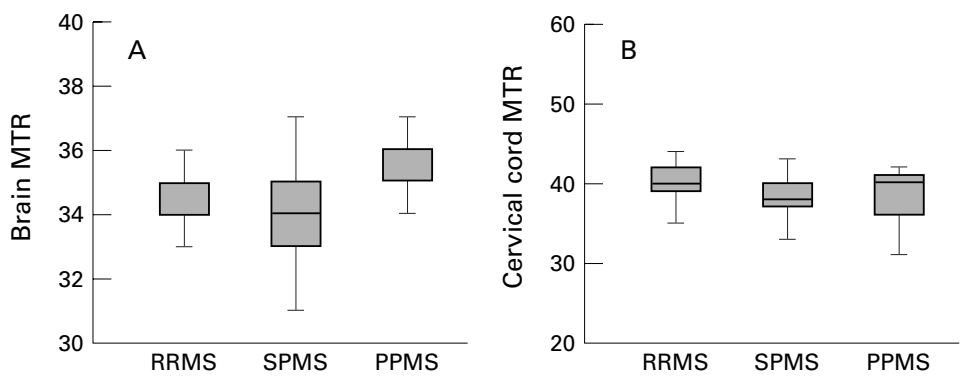

Figure 2 Box plots showing the distribution of brain $(A)$ and cervical cord $(B) M T R$ histogram peak location values from patients with RRMS, SPMS, and PPMS. The boxes represent the interquartile ranges; the horizontal lines within the boxes indicate the median values (for RRMS and PPMS brain histogram peak location, the median values correspond to the lower and upper limits of the box plots, respectively). See text for further details and statistical analysis.
STATISTICAL ANALYSIS

The non-parametric Kruskal-Wallis test was used to compare MRI findings and one way ANOVA was used to compare MTR histogram variables from patients with different clinical phenotypes. At post hoc analysis, the Wilcoxon test and Student's $t$ test for non-paired data were used with Bonferroni correction to adjust for multiple comparisons. Univariate correlations were performed using the Spearman rank correlation coefficient $(r)$. A multivariable logistic model was used to evaluate the effect of brain and cervical cord MTR histogram measures and of brain T2 and T1 LV on the probability of patients having either RRMS or SPMS. The same logistic regression model was applied to assess the effect of these variables on the probability that patients belonged to the groups with or without locomotor disability $(\mathrm{EDSS} \geqslant 4.0 v \mathrm{EDSS}<4.0) .^{26}$

\section{Results}

We studied 77 patients with multiple sclerosis (43 women, $34 \mathrm{men}$ ). Their mean age was 37.5 (SD) 9.3 years, median disease duration was 6 (range 2-34) years, and median EDSS score was 3.0 (range $0.0-7.5$ ). Forty patients were classified as having RRMS (mean age $(\mathrm{SD})=34.0(\mathrm{SD}$ 8.0) years, median disease duration (range) $=4(1-19)$ years, median EDSS score (range) $=1.0(0.0-4.0)), 28$ as SPMS (mean age $(\mathrm{SD})=41.7$ (8.6) years, median disease duration (range) $=10 \quad(4-34)$ years, median EDSS score (range) $=6.0$ (3.07.5)), and nine as PPMS (mean age (SD) $=40.0$ (11.7) years, median disease duration (range) $=8(2-15)$ years, median EDSS score $($ range $)=6.0(3.0-6.5))$.

Brain and cervical cord MRI findings and MTR histogram metrics from the whole patient sample and each of the three multiple sclerosis phenotypes are reported in tables 1 and 2. Brain T1 LV, number and length of cord lesions, and brain and cord MTR histogram peak locations were all significantly different among the three multiple sclerosis phenotypes ( $p$ values ranging from 0.002 to 0.03 ), whereas the difference for brain T2 LV was borderline $(p=0.08)$. At post hoc analysis number and length of cord lesions were significantly higher and brain MTR histogram peak location significantly lower in patients with SPMS than in patients with RRMS and those with PPMS. Cord MTR histogram peak location was also significantly lower in patients with SPMS than in those with RRMS (figs 1 and 2).

Brain T2 LV was modestly correlated with average cord MTR ( $r=-0.2, \mathrm{p}=0.05)$. The univariate correlations between MTR histogram derived metrics obtained from the brain and the cervical cord were all not significant with the exception of that between average brain MTR and cord MTR histogram peak location $(r=0.3, \mathrm{p}=0.008)$.

On a multivariable analysis, both increasing brain T2 LV and decreasing cord MTR histogram peak location values were significantly associated ( $p=0.01$ and 0.02 , respectively) with a higher probability for patients to have SPMS. Odds ratios were 1.05 for T2 LV and 0.81 for 
cord MTR histogram peak location. The same variables were significantly associated $(p=0.006$ and 0.0008 , respectively) with the probability for patients having locomotor disability. Odds ratios were 1.06 for T2 lesion load and 0.77 for cord MTR histogram peak location.

\section{Discussion}

Our results show that the extent and severity of multiple sclerosis pathology in the brain and the cervical cord are associated with the clinical severity of the disease, but the magnitude of the correlation between brain and cervical cord abnormalities is only modest. As a consequence, they indicate that, in multiple sclerosis, MRI and MTI of the brain and cervical cord provide complementary information.

One of the main results of this study is to provide evidence that, in patients with SPMS, several factors contribute to the development of disability. Our results indicate that the accumulation of macroscopic lesions ${ }^{13}{ }^{30}$ and the severity of tissue damage within visible lesions ${ }^{31}$ and in the normal appearing white matter $^{322}$ in the brain and in the cervical cord all contribute to the patients' clinical status. A previous study ${ }^{5}$ with conventional MRI techniques found that patients with SPMS had higher brain hypointense T1 lesion load and more severe cord atrophy than patients with RRMS or PPMS. We also found that patients with RRMS had a less severe macroscopic and microscopic multiple sclerosis pathology than those with SPMS in both the brain and the cervical cord. These results suggest that, even though in patients with RRMS there is a high subclinical disease activity, ${ }^{59}$ this does not lead to severe tissue damage in either of these CNS regions, possibly because of the effectiveness of reparative mechanisms. Interestingly, although brain derived MTR histogram measures were different in patients with SPMS and those with PPMS, there were no significant differences between these two groups for cervical cord MTR histogram metrics. These findings confirm that the extent and severity of microscopic pathology in the cervical cord are similar in the two progressive forms of multiple sclerosis ${ }^{10}$ and may be two of the factors contributing to the progressive accumulation of clinical disability in multiple sclerosis. This is consistent with previous reports of diffuse signal abnormality as a frequent finding on cervical cord T2 weighted scans of patients with PPMS. ${ }^{5}$ Admittedly, the presence of cord atrophy, which is a frequent finding in patients with chronic progressive multiple sclerosis, ${ }^{32} 33$ might also determine a reduction in all the MTR histogram derived measures, as it is more likely that the signal intensity from pixels at the edge of the cord includes a contribution from enlarged CSF spaces. However, we think that our method for the creation of MTR histograms minimised this effect, as, on the one hand, pixels with low signal intensity were excluded from the analysis and, on the other, histograms were normalised for the number of pixels included.
Both brain T2 LV and cervical cord MTR histogram peak location entered a model separating patients with multiple sclerosis with and without locomotor disability, as defined by an EDSS score $\geqslant 4.0$. This score was chosen as a cut off value as higher scores indicate that patients had limited ability to walk, whereas lower values are not related to disability, but to neurological impairment in one or more of the EDSS functional systems. ${ }^{26}$ Although cervical cord damage is more likely to have an impact on locomotor functions, our data indicate that the burden of macroscopic brain T2 lesions explains part of the disability found in our patients. Part of the locomotor disability can be due to multiple sclerosis pathology involving the multiple motor pathways present in the brain. However, as the MTR histogram peak location reflects an increased amount of damaged tissue (the presence of more pixels with lower MTR values), this model implies that the overall extent of macroscopic and microscopic multiple sclerosis pathology in the cervical cord $^{34}$ is also important to determine patients' disability. As a consequence, these data call for an accurate assessment of both brain and spinal cord disease for a better monitoring of multiple sclerosis evolution. Brain T2 LV and cord MTR histogram peak location also fitted the multivariable model, significantly separating patients with RRMS from those with SPMS. This is consistent with the results of the post hoc group comparisons, as the peak location was the only cervical cord MTR histogram derived measure which was significantly lower in SPMS than in RRMS. Moreover, as patients with SPMS had higher EDSS scores than those with RRMS, it is not surprising that the two models gave similar results. Admittedly, as this is a cross sectional study, caution must be exercised before drawing firm conclusions on the multiple sclerosis dynamics and longitudinal studies are warranted to reconsider this issue. Nevertheless, these findings provide insights into the mechanisms leading from a less disabling, relapsingremitting, to a more disabling, chronic progressive course, suggesting that the accumulation of a high burden of multiple sclerosis lesions in the brain can only partially explain this evolution and that severe cervical cord damage is also critical.

The lack of a significant correlation between MRI and MTI measures derived from the brain and the cervical cord seems to indicate that multiple sclerosis abnormalities in these two regions are, at least partially, independent of each other. This is also consistent with the results obtained in previous studies. ${ }^{5910}$ As wallerian degeneration may occur in fibres which go through macroscopic multiple sclerosis lesions, ${ }^{35}$ it might be argued that part of the multiple sclerosis damage in the cord is merely dependent on wallerian degeneration of fibres passing through brain lesions. Previous studies found no ${ }^{10}$ or only very modest ${ }^{5}{ }^{9}$ correlations between the extent of lesions in the brain and the severity of cord pathology, thus suggesting that wallerian degeneration has a minor role in determining cervical cord abnormalities in 
multiple sclerosis. However, as none of these studies quantified the severity of the abnormalities in the brain, the contribution of wallerian degeneration to cervical cord pathology in multiple sclerosis remained unclear. In our study, the poor correlation between MTR histogram derived metrics from the brain and the cervical cord indicates that wallerian degeneration of fibres passing through brain lesions is responsible for only a limited part of the multiple sclerosis pathology in the cord.

We are grateful to $\mathrm{Mr} \mathrm{C}$ Pereira for his assistance in performing MRI. MPS is supported by a grant from TEVA Italy.

1 Ormerod IEC, Miller DH, McDonald WI, et al. The role of NMR imaging in the assessment of multiple sclerosis and isolated neurological lesions: a quantitative study. Brain

2 Rovaris M, Filippi M. Magnetic resonance techniques to monitor disease evolution and treatment trial outcomes in multiple sclerosis. Curr Opin Neurol 1999;12:337-44.

3 Filippi M, Iannucci G, Tortorella C, et al. Comparison of MS clinical phenotypes using conventional and magnetization transfer MRI. Neurology 1999;52:588-94

4 Kidd D, Thorpe JW, Thompson AJ, et al. Spinal cord MRI using multi-array coils and fast spin echo. II. Findings in multiple sclerosis. Neurology 1993;43:2632-7.

5 Lycklama à Nijeholt GJ, van Walderveen MAA, Castelijns JA, et al. Brain and spinal cord abnormalities in multiple sclerosis. Correlation between MRI parameters, clinical sclerosis. Correlation between MRI parameters,
subtypes and symptoms. Brain 1998;121:687-97.

6 Rocca MA, Mastronardo G, Horsfield MA, et al. Comparison of three MR sequences for the detection of cervical son of three MR sequences for the detection of cervical
cord lesions in multiple sclerosis. AfNR Am 7 Neuroradiol cord lesions in mult

7 Stevenson VL, Moseley IF, Phatouros CC, et al. Improved imaging of the spinal cord in multiple sclerosis using threedimensional fast spin echo. Neuroradiology 1998;40:41619.

8 Tartaglino LM, Friedman DP, Flanders AE, et al. Multiple sclerosis in the spinal cord: MR appearance and correlation with clinical parameters. Radiology 1995;195:725-32.

9 Thorpe JW, Kidd D, Moseley IF, et al. Serial gadoliniumenhanced MRI of the brain and spinal cord in early relapsing-remitting multiple sclerosis. Neurology 1996;46:

10 Filippi M, Bozzali M, Horsfield MA, et al. A conventional and magnetization transfer MRI study of the cervical cord in patients with MS. Neurology 2000;54:207-13.

11 Filippi $M$. The role of non-conventional magnetic resonance techniques in monitoring the evolution of multiple sclerosis. 7 Neurol Neurosurg Psychiatry 1998;64(suppl 1):S52-8.

12 Barbosa S, Blumhardt LD, Roberts N, et al. Magnetic resonance relaxation time mapping in multiple sclerosis: normal appearing white matter and the "invisible" lesion load. Magn Reson Med 1994;12:33-42.

13 Truyen L, van Waesberghe JHTM, van Walderveen MAA, al. Accumulation of hypointense lesions (black holes) on T spin-echo MRI correlates with disease progression in multiple sclerosis. Neurology 1996;47:1469-76.

14 van Walderveen MAA, Kamphorst W, Scheltens $\mathrm{P}$, et al. Histopathologic correlate of hypointense lesions on T1 weighted spin-echo MR in multiple sclerosis. Neurology 1998;50:1282-8.

15 McGowan JC, Filippi M, Campi A, et al. Magnetisation transfer imaging: theory and application to multiple sclerosis. $\mathcal{F}$ Neurol Neurosurg Psychiatry 1998;64(suppl 1):S66-9.
16 Dousset V, Brochet B, Vital A, et al. Lysolecithin-induced demyelination in primates: preliminary in vivo study with $\mathrm{MR}$ and magnetization transfer. AfNR Am $\mathcal{f}$ Neuroradiol 1995;16:225-31.

17 Dousset V, Grossman RI, Ramer KN, et al. Experimental allergic encephalomyelitis and multiple sclerosis: lesion characterization with magnetization transfer imaging. Radiology 1992;182:483-91.

18 Filippi M, Campi A, Dousset V, et al. A magnetization transfer imaging study of normal-appearing white matter in multiple sclerosis. Neurology 1995;45:478-82.

19 van Waesberghe JH, Kamphorst W, De Groot CJ, et al. Axonal loss in multiple sclerosis lesions: magnetic resonance imaging insights into substrates of disability. Ann Neurol 1999;46:747-54.

20 van Buchem MA, McGowan JC, Kolson DL, et al. Quantitative volumetric magnetization transfer analysis in multiple sclerosis: estimation of macroscopic and microscopic disease burden. Magn Reson Med 1996;36:632-6.

21 Rovaris M, Filippi M, Falautano M, et al. Relation between MR abnormalities and patterns of cognitive impairment in multiple sclerosis. Neurology 1998;50:1601-8.

22 Tortorella C, Viti B, Bozzali M, et al. A magnetization transfer histogram study of normal appearing brain tissue in multiple sclerosis. Neurology 2000;54:186-93.

23 van Buchem MA, Grossman RI, Armstrong C, et al. Correlation of volumetric magnetization transfer imaging with clinical data in MS. Neurology 1998;50:1609-17.

24 Poser CM, Paty DW, Scheinberg L, et al. New diagnostic criteria for multiple sclerosis: guidelines for research protocols. Ann Neurol 1983;13:227-31.

25 Lublin FD, Reingold SC, the National MS Society (USA) Advisory Committee on Clinical Trials of New Agents in Multiple Sclerosis. Defining the clinical course of multiple sclerosis: results of an international survey. Neurology 1996; 46:907-11

26 Kurtzke JF. Rating neurological impairment in multiple sclerosis: an expanded disability status scale (EDSS). Neurology 1983;33:1444-52.

27 Miller DH, Barkhof F, Berry I, et al. Magnetic resonance imaging in monitoring the treatment of multiple sclerosis: concerted action guidelines. 7 Neurol Neurosurg Psychiatry 1991;54:683-8

28 Rovaris M, Filippi M, Calori G, et al. Intra-observer reproducibility in measuring new putative markers of demyelination and axonal loss in multiple sclerosis: a comparison with conventional $\mathrm{T}_{2}$-weighted images. F Neurol 1997;244: 266-70.

29 Studholme C, Hill DLG, Hawkes DJ. Automated 3D registration of MR and PET brain images by multi-resolution optimisation of voxel similarity measures. Med Physics 1997;24:25-35.

30 Thompson AJ, Kermode AG, Wicks D, et al. Major differences in the dynamics of primary and secondary progressive multiple sclerosis. Ann Neurol 1991;29:53-62.

31 Rocca MA, Mastronardo G, Rodegher M, et al. Long-term changes of magnetization transfer-derived measures from patients with relapsing-remitting and secondary progressive multiple sclerosis. AfNR Am f Neuroradiol 1999;20: $821-7$.

32 Filippi M, Campi A, Colombo B, et al. A spinal cord MRI study of benign and secondary progressive multiple sclerosis. F Neurol 1996;243:502-5.

33 Stevenson VL, Leary SM, Losseff NA, et al. Spinal cord atrophy and disability in MS. A longitudinal study. Neurology 1998;51:234-8.

34 Lovas G, Szilagyi N, Majtenyi K, et al. Axonal changes in chronic demyelinated cervical spinal cord plaques. Brain 2000;123:308-17.

35 De Stefano N, Narayanan S, Matthews PM, et al. In vivo evidence for axonal dysfunction remote from focal cerebral demyelination of the type seen in multiple sclerosis. Brain 1999;122:1933-9. 